\title{
ERRATUM TO "FORMULAS AND THEOREMS FOR THE SPECIAL FUNCTIONS OF MATHEMATICAL PHYSICS" BY W. MAGNUS, F. OBERHETTINGER, R. P. SONI
}

\author{
RADOSŁAW SZMYTKOWSKI
}

\begin{abstract}
We correct 25 errors and misprints detected in "Formulas and Theorems for the Special Functions of Mathematical Physics", third edition, by W. Magnus, F. Oberhettinger, R. P. Soni (Springer, Berlin, 1966), and not listed in earlier errata for this handbook published in Mathematics of Computation.
\end{abstract}

The following errors and misprints, not listed in earlier errata for this handbook [Math. Comput. 23 (1969) 471; 24 (1970) 240; 24 (1970) 505; 25 (1971) 201; 29 (1975) 672; 30 (1976) 677-8; 32 (1978) 319-20; 36 (1981) 315-7; 41 (1983) 775-8], have been detected (see next page):

Received by the editor December 28, 2011.

2010 Mathematics Subject Classification. Primary 33-00. 


\begin{tabular}{|c|c|c|}
\hline Location & For & Read \\
\hline $\begin{array}{l}\text { page } 54, \text { line } 2 \\
\text { page } 168, \text { line } 1 \text { up }\end{array}$ & $\begin{array}{l}P\left(-\frac{\sqrt{x}}{\sqrt{1+x}}\right) \\
(1+\nu+\mu)\end{array}$ & $\begin{array}{l}P_{a+b-1}^{b-a}\left(-\frac{\sqrt{x}}{\sqrt{1+x}}\right) \\
(1+\nu+\mu)_{l}\end{array}$ \\
\hline page 170 , line 12 & $0<x<1$ & $-1<x<1$ \\
\hline page 171, line 4 & $\Gamma\left(1+\frac{1}{2} \nu-\frac{1}{2} \mu\right)$ & $\Gamma\left(\frac{1}{2}+\frac{1}{2} \nu-\frac{1}{2} \mu\right)$ \\
\hline page 172 , line 7 up & $\left.1-x^{2}\right)^{-\frac{1}{4}}$ & $\left(1-x^{2}\right)^{-\frac{1}{4}}$ \\
\hline page 178 , line 6 & $\frac{\partial}{\partial v}$ & $\frac{\partial}{\partial \nu}$ \\
\hline page 183 , line 2 & $x<\cos \varphi$ & $\cos \varphi<x<1$ \\
\hline $\left.\begin{array}{l}\text { page } 197, \\
\text { entry for } P_{\nu}(x), \\
\text { the third column }\end{array}\right\}$ & $\gamma$ & $2 \gamma$ \\
\hline $\left.\begin{array}{l}\text { page } 197, \\
\text { entry for } Q_{\nu}(x), \\
\text { the third column }\end{array}\right\}$ & $\gamma$ & $2 \gamma$ \\
\hline page 200 , line 7 up & $\times F$ & $\times z^{-\alpha-2 \nu} F$ \\
\hline page 200 , line 7 up & $z^{2}$ & $z^{-2}$ \\
\hline page 219, line 7 & $x^{2}-1$ & $1-x^{2}$ \\
\hline page 260 , line 10 & $y=\frac{1}{\sqrt{1-x^{2}}} T_{n}(x)=U_{n-1}(x)$ & $y_{1}=\frac{T_{n}(x)}{\sqrt{1-x^{2}}}, y_{2}=U_{n-1}(x)$ \\
\hline page 298 , line 2 & $\Gamma(1+2 \mu)$ & $\Gamma(1-2 \mu)$ \\
\hline page 298 , line 2 & $M_{k,-\mu}(z)$ & $M_{\varkappa,-\mu}(z)$ \\
\hline page 302 , line 5 up & $\sqrt{z} W_{\varkappa, \mu}^{\prime}(z)$ & $2 \sqrt{z} W_{\varkappa, \mu}^{\prime}(z)$ \\
\hline page 302 , line 4 up & $\sqrt{z} W_{\varkappa, \mu}^{\prime}(z)$ & $2 \sqrt{z} W_{\varkappa, \mu}^{\prime}(z)$ \\
\hline $\begin{array}{l}\text { page } 302, \text { line } 2 \text { up } \\
\text { page } 303, \text { line } 6\end{array}$ & $\begin{array}{l}2 W^{\prime} \varkappa+\frac{1}{2}, \mu+\frac{1}{2} \\
(1+\mu) \\
(1+2 \varkappa)\end{array}$ & $\begin{array}{l}W^{\prime} \\
\quad \varkappa+\frac{1}{2}, \mu+\frac{1}{2}(z) \\
(1+2 \mu-2 \varkappa)\end{array}$ \\
\hline page 305 , line 3 & $e^{-i \frac{\mu}{4}(2 \mu-1)}$ & $e^{-i \frac{\pi}{4}(2 \mu-1)}$ \\
\hline page 305 , line 3 & $J_{\mu}\left(-\frac{1}{2} z\right)$ & $J_{\mu}\left(\frac{1}{2} z e^{i \pi}\right)$ \\
\hline page 317 , line 11 & $\Gamma\left(\frac{1}{2}+\mu-\varkappa\right)$ & $\Gamma\left(\frac{1}{2}+\mu+\varkappa\right)$ \\
\hline page 317 , line 11 & $e^{i \pm \frac{\pi}{2}(2 \varkappa-2 \mu-1)}$ & $e^{ \pm i \frac{\pi}{2}(2 \varkappa-2 \mu-1)}$ \\
\hline page 324, line 2 & $\begin{array}{l}\left(\nu+\frac{1}{2}-\frac{1}{4} z^{2}\right) \\
\frac{\sqrt{2 \pi}}{\Gamma(1+\nu)}\end{array}$ & $\begin{array}{l}\left(\nu+\frac{1}{2}-\frac{1}{4} z^{2}\right) y \\
\frac{i \sqrt{2 \pi}}{\Gamma(1+\nu)}\end{array}$ \\
\hline
\end{tabular}

Atomic Physics Division, Department of Atomic Physics and Luminescence, Faculty of Applied Physics and Mathematics, Gdańsk University of Technology, Narutowicza 11/12, 80-233 GDAŃsk, POLAND

E-mail address: radek@mif.pg.gda.pl 